\title{
Rhombencephalosynapsis, The Famous Unknown: Own Experience and Literature Review of Prenatal Diagnosis with Sonography and Magnetic Resonance Imaging
}

\author{
Spinelli $\mathbf{M}^{1,2 *}$, Vascone $\mathrm{C}^{2}$, Meglio $\mathbf{L D}^{2}$, Sica $\mathrm{C}^{2}$, \\ Raio $\mathrm{L}^{1}$ and Meglio $\mathrm{AD}^{2}$ \\ ${ }^{1}$ Department of Obstetrics and Gynecology, University of \\ Bern, Bern, Switzerland \\ ${ }^{2}$ Private Centre "Diagnostica Ecografica Prenatale Aniello \\ Di Meglio srl”, Naples, Italy \\ *Corresponding author: Marialuigia Spinelli, \\ Department of Obstetrics and Gynecology, \\ Friedbuhlstrasse 19, University of Bern, 3010 Bern, \\ Switzerland
}

Received: April 22, 2021; Accepted: May 18, 2021; Published: May 25, 2021

\begin{abstract}
Rhombencephalosynapsis (RES) is a rare cerebellar malformation characterized by congenital fusion of the hemispheres and absence of the vermis. This condition is associated with developmental delay, seizures and involuntary head movements. Although the clinical and imaging aspect of this condition have been thoroughly investigated in the adult, prenatal diagnosis remains still a challenge in the modern Fetal-Maternal Medicine. Here we report our experience with 3 cases and review the current literature as well, focusing specifically on the obstetric imaging as well as on the prenatal diagnosis and management of this rare condition. RES should be considered in the differential diagnosis when absence of the vermis in the Posterior Fossa (PF) is suspected at prenatal Ultrasound Sonography (US), especially when ventriculomegaly or other Central Nervous System (CNS) anomalies are detected. A complete anatomical workup is necessary in these cases. Magnetic Resonance Imaging (MRI) remains to be the imaging modality of choice in confirming the diagnosis.
\end{abstract}

Keywords: Rhomboencephalosynapsis; Prenatal diagnosis; Ultrasound; Magnetic resonance imaging; Cerebellum; vermis

\section{Background}

Rhomboencephalosynapsis (RES) is a rare congenital malformation defined by vermian agenesis, fusion of the cerebellar hemispheres and apposition or fusion of the dentate nuclei [1]. Most of the reported cases are associated with supra-tentorial Central Nervous System (CNS) anomalies; however, a few cases present as an isolated cerebellar malformation $[2,3]$.

RES produces severe motor and intellectual developmental delay when it is associated with other CNS anomalies. However, also when RES is an isolated finding, major psychiatric disorders such as obsessive oral self-mutilation have been reported [4]. Therefore, prenatal diagnosis of RES, both associated with other anomalies and isolated, represents an important issue for prenatal Medicine.

Because there is still little information about prenatal diagnosis of RES [3], the aims of this paper were (a) to report our experience with RES, presenting 3 cases in which prenatal two-dimensional ultrasound scan (2D-US) and intrauterine Magnetic Resonance Imaging (MRI) clearly depicted cerebellar features characteristic of RES; (b) to review the existing literature referring to prenatal diagnosis of RES.

\section{Data Sources}

\section{Own experience}

Our experience includes 3 fetuses examined at the Gestational Age (GA) of 24, 25 and 27 weeks at prenatal 2D-US from January 2013 to January 2016. The cases were retrospectively reviewed including ultrasound pictures, MRI data and medical records. The ultrasound machine used in all cases was standard Aloka (Aloka Co., Ltd, Tokyo, Japan) equipped with a curved linear array transabdominal transducer $(2-5 \mathrm{MHz})$.

All of them underwent intrauterine MRI, performed at 1.5T using the following technique: multiplanar 3- to 4-mm thick, single-shot fast spin echo, T2 weighted sections (repetition time/echo time = $3000 / 90$ milliseconds, field of view $=340 \mathrm{~mm}$, matrix $=2563192)$.

Two out of 3 cases underwent molecular karyotype analysis with CGH array.

Our report is in accordance with the Declaration of Helsinki and was approved by the Review Board of the Clinical Research of the University of Bern. All adult participants provided written informed consent to participate in this report.

\section{Literature review}

We searched articles published without language limitation and identified via a search of MEDLINE and EMBASE, from 1990 to date, following medical subject heading terms: "Rhomboencephalosynapsis", "Vermian agenesis", "Vermian hypoplasia", "Cerebellar hypoplasia" and "Prenatal diagnosis". Only studies reporting prenatal diagnosis of RES were selected. All crossreferences were hand-searched. All types of studies were selected.

\section{Results}

\section{Own experience}

Case 1: A 33-year-old woman, gravida 2, para 0 (previous miscarriage during the second trimester), was referred to our Centre because a previous 23-week-GA US had revealed a tri-ventricular hydrocephaly. The karyotype with CGH array was normal (46XX). Our 24-week transabdominal US confirmed these findings and revealed an abnormal Posterior Fossa (PF), with a severe reduction of
Annals Thyroid Res - Volume 7 Issue 3 - 2021

Submit your Manuscript | www.austinpublishinggroup.com

Spinelli et al. () All rights are reserved
Citation: Spinelli M, Vascone C, Meglio LD, Sica C, Raio L and Meglio AD. Rhombencephalosynapsis, The Famous Unknown: Own Experience and Literature Review of Prenatal Diagnosis with Sonography and Magnetic Resonance Imaging. Annals Thyroid Res. 2021; 7(3): 342-348. 

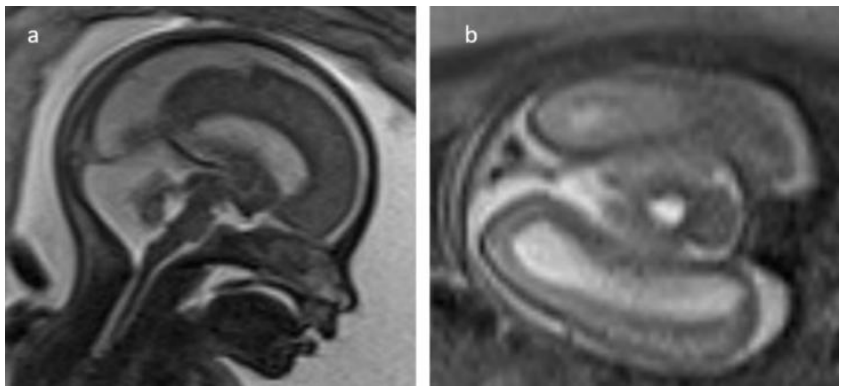

Figure 1: Case 1: Intrauterine MRI. Cerebellar hypoplasia, with rhomboencephalosynapsis. Hydrocephalus. FSE/T2-weighted images. (a) Midsagittal plane; (b) Axial plane.

the trans-cerebellar diameter below the fifth centile. The vermis was not clearly visualized. A 25-week intrauterine MRI was performed, showing agenesis of the cerebellar vermis, dorsal fusion of the cerebellar hemispheres, with confirmation of the significant reduction of the cerebellar transverse diameter. These features, together with the "keyhole" shape appearance of the fourth ventricle, were consistent with those already well known from postnatal MRI studies of RES. A tri-ventricular ventriculomegaly, with $19 \mathrm{~mm}$ atrial width, was also evident (Figure 1). The septum pellucidum and corpus callosum were visible. The brain parenchyma appeared normal. The patient decided for termination without fetal autopsies and then was lost at follow-up.

Case 2: A 31-year-old woman, gravida 3, para 2 (her previous pregnancies was uneventful, with normal-term deliveries), presented at our Centre at 25-weeks of GA, because a previous scan at 24 weeks had revealed a renal disease. At our US, the fetal kidneys were symmetrically enlarged (right, $5.3 \times 4.0 \mathrm{~cm}$; left, $4.3 \times 3.7 \mathrm{~cm}$ ) and highly echogenic, leading to the absence of the corticomedullary differentiation, suspicious of Autosomal Dominant Polycystic Kidney Disease (ADPKD). Checking the kidneys of both the parents,

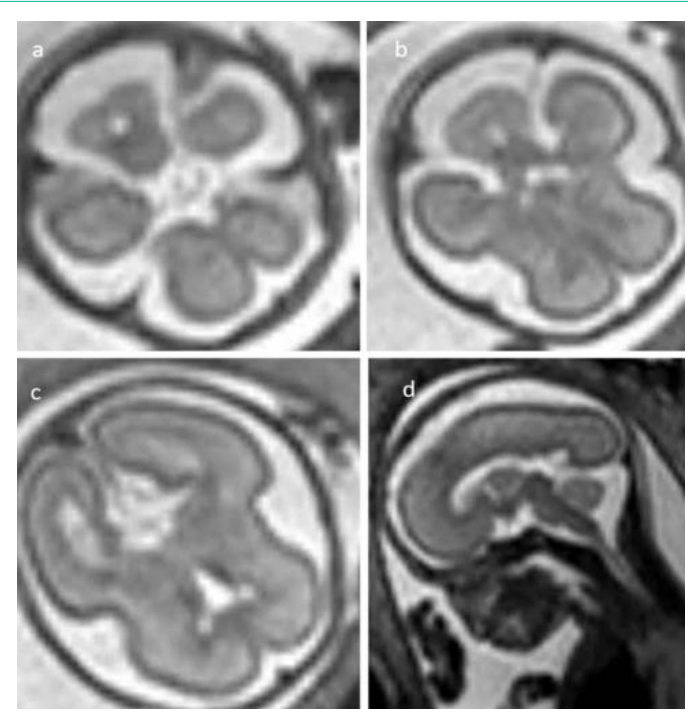

Figure 2: Case 2: Intrauterine MRI. Agenesis of the cerebellar vermis, dorsal fusion of the cerebellar hemispheres, which appears without the normal cerebellar sulci, and a reduction of the fourth ventricle. No other brain anomalies. FSE/T2-weighted images. (a, b, c) Axial plane; (d) Mid-sagittal plane. we did not find any renal US abnormality. When we scanned the fetal brain, an abnormal PF was evident, with cerebellar hypoplasia and an abnormal shape of the cerebellar hemispheres. The vermis was not clearly depicted. No other brain anomalies were detected. Investigating family history of congenital malformations, the woman revealed that the first daughter of 3 years old had been diagnosed postnatally with ventriculomegaly and had cognitive impairment as well as a coloboma of the optic nerve. The karyotype of this first daughter was normal. In the current pregnancy, the karyotype with CGH array was normal too (46XY). At 26 weeks, a prenatal MRI examination was performed, showing agenesis of the cerebellar vermis, dorsal fusion of the cerebellar hemispheres, which appeared without the normal cerebellar sulci, and a reduction of the fourth ventricle (Figure 2). These signs were compatible with RES. The parents decided to terminate the pregnancy at another institution.

Case 3: A 42-year-old woman, gravida 1, para 0, was referred to our Centre at 26 weeks of GA because of a vaginal bleeding. When we scanned the fetal brain, an abnormal PF was evident, with absent visualization of the vermis, fusion of the cerebellar hemispheres with a reduction of the trans-cerebellar diameter $(22 \mathrm{~mm}$, below the fifth percentile), without demonstration of any communication between the fourth ventricle and the cisterna magna. Diagnosis of RES was thus formulated (Figure 3). No other Central Nervous System (CNS) and extra-CNS fetal anomalies were detected. A 27-week GA intrauterine MRI scan was performed, confirming the cerebellar transverse diameter reduction, the agenesis of the vermis, as well as the dorsal fusion of the cerebellar hemispheres, consistent with RES. At 32 weeks GA, a preterm vaginal delivery occurred, which resulted in a stillborn female baby. The woman did not authorize autopsy nor any other postnatal investigation.

\section{Literature review}

Of the 186 articles related to our key words, 83 were excluded after reading the titles because they were not pertinent to RES. Among the 103 remaining articles, 92 were excluded after reading the abstracts because they did not refer to prenatal diagnosis. The other 11 articles were selected for our review. Two additional papers were added by searching cross-references (i.e., reading all references in the included articles). Thus, 13 articles including case reports, case series and conference proceedings were finally analyzed.

Since 1990, according to our research criteria, 65 cases of prenatal

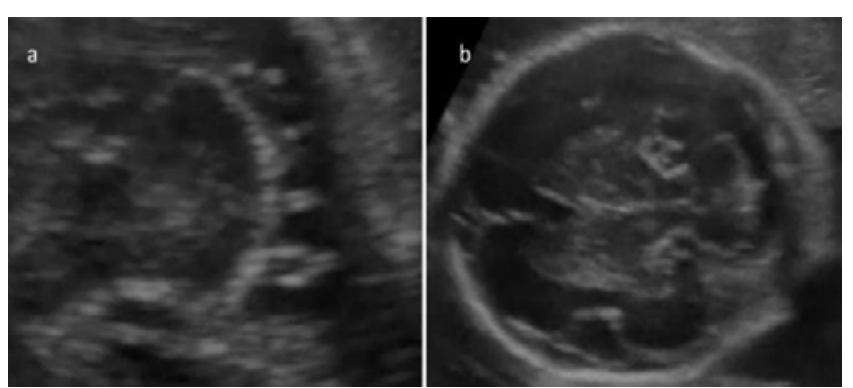

Figure 3: Case 3: Prenatal US. Absent visualization of the vermis, fusion of the cerebellar hemispheres with a reduction of the trans-cerebellar diameter below the fifth centiles, without demonstration of any communication between the fourth ventricle and the cisterna magna. No other brain anomalies. Twodimensional ultrasound scans. (a) Axial plane, with magnification of the posterior fossa; (b) Axial plane. 
Table 1: List of case reports of Rhomboencephalosynapsis (RES) diagnosed prenatally (NA: Not Available; CS: Caesarean Section; T: Termination of pregnancy; VD: Vaginal Delivery: wks: weeks).

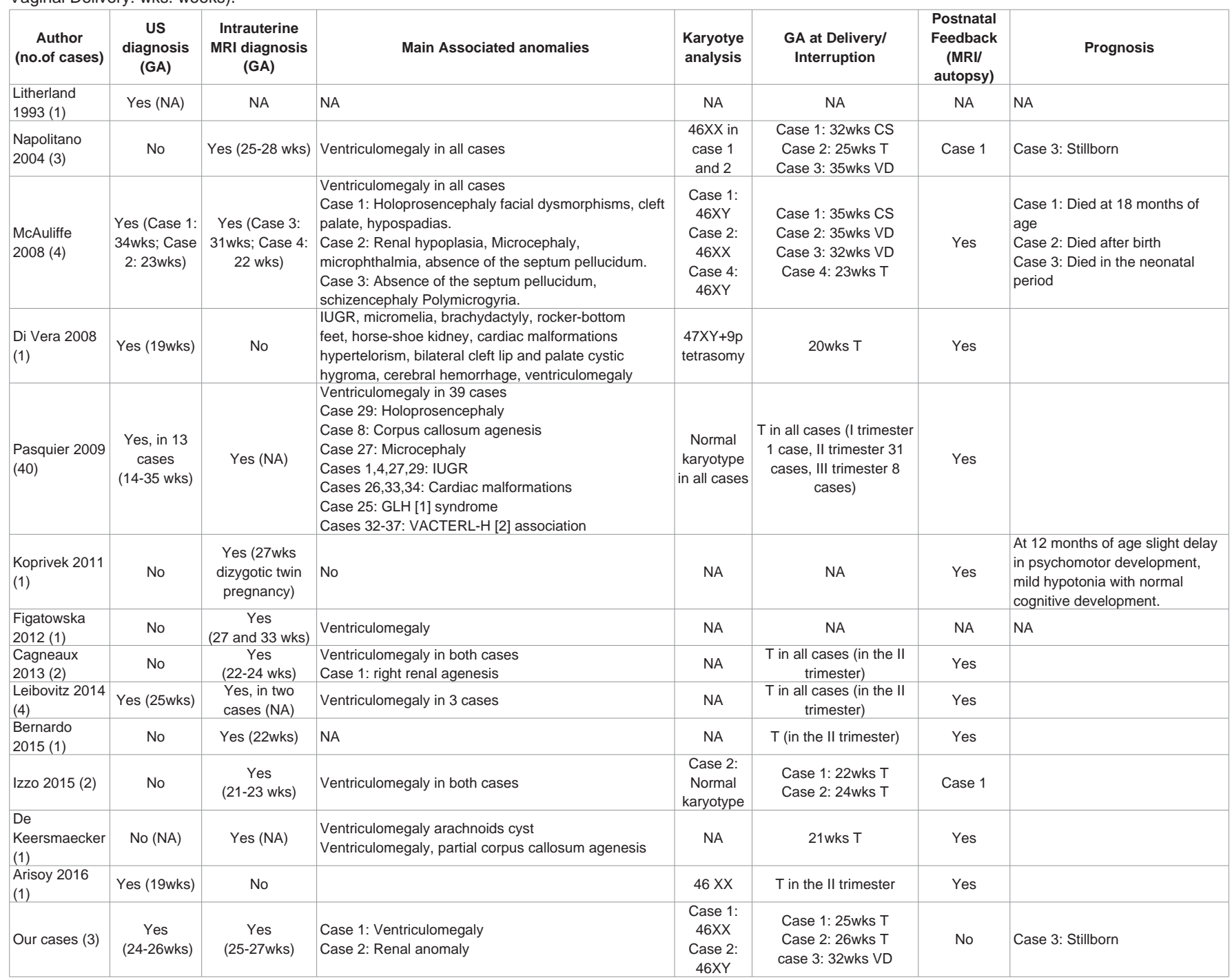

[1] Gomez Lopez Hernandez.

[2] Vertebral anomalies, Anal atresia, Cardiovascular anomalies, Trachea-oesophageal fistula, Renal anomalies, Limb defects.

diagnosis of RES have been reported, including the ours. In 25 cases (38\%), diagnosis was first formulated or strongly suggested at prenatal US during the second trimester of pregnancy; however in 64 cases (97\%) a definitive diagnosis was performed at intrauterine MRI. Mean \pm SD (range) GA at US and MRI diagnosis were 23.86 \pm 5.14 (14-35) and 24.92 \pm 2.9 (21-31), respectively. Documented associated anomalies were present in 60 cases $(91 \%)$. Mean \pm SD GA at delivery or at termination were $33.71 \pm 1.60$ (32-35) and 24.92 \pm 2.9 (21-31), respectively. A postnatal feedback with MRI andlor autopsies was available in 57 cases (88\%). Characteristic of the included studies are listed in Table 1.

\section{Discussion}

\section{Epidemiology, embryopathogenesis and etiologic factors}

RES was first described by Obersteiner from the post-mortem examination of a 28-year-old man who committed suicide [5]. This condition is characterized by vermian agenesis associated with fusion of the cerebellar hemispheres, which have a rhomboid shape, fusion of peduncles, and of the dentate nuclei. Kinking of the brainstem is often present and the PF appears smaller than normal [6-17]. Its frequency has been estimated at $0.13 \%$ by Sener from a series of 3000 consecutive pediatric brain MRIs [18].

In the traditional view of embryonic development, the cerebellum arises from 2 distinct embryonic primordia known as the rhombic lips [10]. The vermis arises superiorly between these two embryonic cerebelli. At the beginning of 8 weeks of gestation, proliferating neuroblasts from symmetric alar plates form paired rhombic lips that thicken, project into the fourth ventricle, and extend progressively toward the midline. At the end of 12 weeks of gestation, the rhombic lips on the 2 sides fuse in the midline beginning rostrally; the anterior vermis is thereby formed before the posterior vermis $[10,15]$. The absent vermis results in abnormal cerebellar hemispheric fusion, malorientation of folia, and a horseshoe-shaped dentate nucleus around a teardrop-shaped fourth ventricle [1]. The proposed embryologic schema accounts for the transverse orientation of the cerebellar folia, dentate nuclei, and varying cerebellar peduncular 
fusions as well as for the absence of the olivary nuclei.

Another view of embryonic development supported by Sidman et al. [19] is that the cerebellar primordium arises from the tuberculum cerebelli, a band of tissue in the dorsal part of the first neuromere that crosses the midline in the shape of an inverted V. They suggest that the cerebellar primordium is unpaired and that on transverse section, it might be misinterpreted as a pair of symmetric but unconnected structures, the rhombic lips.

According to this last embryologic view, RES results from failure in vermian differentiation of the more rostral part of the midline primordium; the fused cerebellar hemispheres may be explained by the fact that the cerebellar primordium is unpaired [19-23]. The cause of this failure of differentiation is still unclear. Some studies have pointed out a genetic defect in the isthmic organizer, resulting in abnormal dorsal patterning [24]. FGF8 and Lmxla have been suggested as possible candidate genes [25].

RES seems to be sporadic, without any instance of familial recurrence. The possibility of autosomal recessive inheritance was reported in only 1 patient [15]. Our review of prenatal cases confirms these post-natal data, with no case of any familiarity of this condition. In case 2 of our series, it has not been confirmed any relation between the condition of the fetus and that of the first daughter of the pregnant woman.

No teratogenic factors have been recorded, with the exception of 1 post-natal case associated with maternal phencyclidine abuse [20] and another associated with maternal ethosuximide medication [13]. Among the cases included in our review, McAuliffe [26] reported a maternal history of recreational drug use in the first trimester in a case of RES. No teratogenic factors have been recorded in our case series.

\section{Prenatal diagnosis}

Because of the rarity of the condition, no data are available about frequency of prenatal diagnosis. Our review showed 65 cases of RES diagnosed prenatally, with 61 (94\%) detected during the last 10 years. Among these, two cases (case 1 and 2 of McAuliffe [26]) were strongly suspected prenatally but definitively established postnatally.

Our 3 cases were recorded over a 3-year-observation. The relatively high incidence of RES in our personal experience may be explained by the fact that our facility is a tertiary care Centre for prenatal diagnosis, where every year more than 4000 pregnancies are examined.

The diagnosis at prenatal imaging is established with the identification of a hypoplastic single-lobed cerebellum. This can be visualized by intrauterine MRI and, recently, also by US, as showed in our review [17,26-37]. Coronal and axial planes are superior for this diagnosis and sagittal views are least helpful.

Coronal MRI findings include the visualization of fused cerebellar hemispheres with absent or severely hypoplastic vermis and an absent vallecula. A narrow diamond-shaped fourth ventricle and a fused horseshoe-shaped dentate nucleus are the features seen on axial MRI planes. Sagittal views are remarkable for the lack of visualization of the primary vermian fissure and lack of a normal fastigial point of the fourth ventricle [17,26-37].
The prenatal US features of RES are a small cerebellum and small or absent vermis. With vertex presentation, the addition of transvaginal to transabdominal US may allow better visualization of the PF and should be considered when the intracranial anatomy appears abnormal [38]. The postero-inferior aspect of the vermis may remain open until 17.5 week - GA [39]; this needs to be taken into consideration when examining the PF at or before 18 week - GA.

The main differential diagnosis of small or absent vermis at prenatal imaging includes Dandy-Walker (DWM) and Chiari II malformation [29]. In contrast to RES, in the first condition the vermian maldevelopment is characterized by complete or partial agenesis, cystic dilatation of the fourth ventricle and enlarged PF with upward displacement of the tentorium; while, the second is associated with an open spinal dysraphism and obliteration of the cisterna magna, as well as with abnormal curvature of the cerebellar hemispheres (the "banana sign") [21,22].

Our review showed that $38 \%$ of diagnoses were formulated or at least strongly suggested at prenatal US during the second trimester of pregnancy, with a likely increasing detection rate over time. However, $97 \%$ of definitive diagnoses were performed at intrauterine MRI. In our series, in case 3 RES was clearly diagnosed at US with confirmation by MRI, while, in the other 2 cases, RES was strongly suspected at US time, but diagnosis was made at MRI.

It is remarkable that in $92 \%$ of prenatal US diagnoses, other CNS - abnormalities were associated to RES, with the exception of case 2 and 3 of our series. These data may show that prenatal US may be accurately able to depict RES in the fetus especially when other CNS anomalies are present. In our opinion, this may be explained not only by the epidemiological data, which show the high frequency of associated CNS-abnormalities [1-10,21-23] in RES, but also by the fact that the presence of any associated CNS-abnormality, detectable at prenatal US, may play the role of an "alarm bell", thus inducing the clinician to a deeper and a more careful study of the whole CNS, including the PF.

Conversely, the only case of an isolated prenatally- detected RES included in our review was diagnosed exclusively at MRI [28]. These data show that intrauterine MRI remains the diagnostic tool that allows the unequivocal diagnosis of RES especially in cases of an isolated finding [28]. In this regard, it is remarkable that in our series, 2 out of 3 cases of RES showed no other associated brain anomalies and the indications for US examination were related to issues not involving the CNS, i.e. the renal anomalies (case 2), as well as the vaginal bleeding (case 3).

\section{Associated non-chromosomal abnormalities}

RES is frequently associated with other CNS- and extra CNS abnormalities [1-10,21-23].

Associated CNS- supra-tentorial abnormalities include hydrocephalus or ventriculomegaly; absence of the septum pellucidum, and Agenesis of the Corpus Callosum (ACC) or corpus callosum anomalies, usually described as thin, stretched, or angulated [12-17]. Additional anomalies include: fusion of the thalami, tectum, and fornices; hypoplastic chiasm and underdeveloped temporal lobes [1]; fusion of the quadrigeminal plate and middle and superior cerebellar peduncles; hypoplastic olivary nuclei [12]; 
hypoplastic anterior commissure and temporal lobes [9]; cleft in the parieto-occipital lobe and frontal encephalocele [13]; small occipital encephalocele; and multiple subcortical and periventricular heterotopias [7].

Extra-CNS associated - anomalies have been reported too, including segmentation and fusion anomalies of the spine and musculoskeletal-anomalies, especially phalangeal and radial ray defects [2]. Associated defects of the cardio vascular, respiratory and urinary tract have also been reported $[17,26]$

Our survey is in accordance with the available literature, showing that anomalies associated to RES were present in more than $90 \%$ of cases, with ventriculomegaly representing the most frequent associated malformation (86\% of cases), including 1 out of 3 of our cases (case 1). The most common extra CNS - associated malformation involve the urinary apparatus ( $20 \%$ of cases with extraCNS associated abnormalities), detected also in one of our cases (case 2). In this our case, the fetal kidneys were symmetrically enlarged and highly echogenic, leading to the absence of the corticomedullary differentiation, suspicious of ADPKD. The association of RES with ADPKD has been already reported in the literature [40]. Unfortunately, we could not confirm this suspicious postnatally.

Pasquier et al. [17] reported, in morphological analysis of 40 fetuses after medical termination of pregnancy, that RES was associated with ventriculomegaly, ACC, lobar holoprosencephaly (HPE) and microcephaly as others CNS - anomalies; cleft palate, thymic hypoplasia, hypothophy, cardiac anomalies, genito-urinary malformations (renal agenesis, hemiuterus) and vertebral defects as extra CNS. They also reported that, except for only one case, all fetuses presented with macrocranium with large fontanels and a characteristic, although not specific, cranio-facial dysmorphism, associating prominent forehead, hypertelorism, small pointed nose with a large nasal bridge and anteverted nostrils, midface hypoplasia, smooth philtrum, small mouth with thin lips, microretrognathism as well as posteriorly rotated low set ears with a coarse up-sized lobule and deformation of the external auditory canal. In addition, Gomez Lopez Hernandez (GLH) syndrome in one case $(2.5 \%)$ and VACTERL-H ('Vertebral anomalies, Anal atresia, Cardiovascular anomalies, Trachea-oesophageal fistula, Renal anomalies, Limb defects') association in 6 cases (15\%) were identified [17]. GLH syndrome is characterized by the triad of RES, trigeminal anesthesia and bilateral parieto-occipital alopecia with typical craniofacial anomaly including hypertelorism, brachyturricephaly and midface retrusion and a short stature [41]. In this regard, some authors suggested that if a diagnosis of RES is made, GLH syndrome and VACTERL association should be excluded [41]. In our cases, RES was associated with hydrocephalus (case 1) and renal anomalies (case 2) but not with other malformations suggestive of any syndromic manifestation.

The postnatal evaluation of case 1 of McAuliffe [26] revealed RES with ventriculomegaly and lobar HPE by postnatal MRI. Moreover, polygyria, aqueduct stenosis, cleft palate, absence of thymus, hypoplasia of thyroid, inguinal hernia, absent left kidney and facial dismorphism were detected at autopsy. In the postnatal evaluation of case 2, RES with ventriculomegaly was detected by postnatal MRI, together with microcephaly, midfacial hypoplasia, microphthalmia, short webbed neck, duodenal atresia, absent right kidney and left single palmar crease. They also reported that in case 3 HPE, ACC, schizencephaly and polygyria were associated with RES. In addition, in case 4, aquaductal stenosis and RES were determined in autopsy [26].

Di Vera (2008) showed that RES was associated with micromelia, brachydactyly, rocker-bottom feet, horse-shoe kidney, atrioventricular septal defect, truncus arteriosus, hypertelorism, bilateral cleft lip and palate with pre-maxillary protrusion, cystic hygroma, pre-auricular tag, cupped ears, cerebral haemorrhage and bilateral borderline ventriculomegaly (di Vera et al. 2008).

Rarely, RES is an isolated malformation. In our review, only the case of Koprivek [28] and our case 3 were isolated. These authors [28] reported the case of 27 weeks - GA fetus in a dizygotic twin pregnancy with partial RES. In our case series, case 3 was casually detected in a 26 week - pregnant woman undergoing US for a vaginal bleeding. The other few cases of an isolated RES reported in the literature have been detected postnatally [42].

\section{Associated chromosomal abnormalities}

While the risk of non-chromosomal malformations in RES is high, conversely the risk of chromosomal abnormalities is low. The results of chromosomal analyses performed in previous cases diagnosed postnatally were normal except for an interstitial deletion of chromosome 2q.1 [1].

The results of our review of prenatally - diagnosed cases are consistent with the previous ones, showing that $98 \%$ of cases, in which karyotype analysis was performed/available, had normal karyotype without any gender prevalence.

Di Vera [32] reported RES with tetrasomy 9p. Tetrasomy 9p was first described by Ghymers in 1973 [43]. Since then few cases have been reported and a review of previously reported features described the variability of appearance in fetuses and newborns affected by tetrasomy $9 \mathrm{p}$ mosaic and non-mosaic [44]. Fetuses with tetrasomy $9 p$ that includes the region 9pterp11.1 are likely to present a DWM and ventriculomegaly on prenatal ultrasound [45]. So far, this case of di Vera [32] was the first report of RES in a fetus with tetrasomy $9 p$ deriving from assisted reproduction technologies (ARTs). In our report, cases 1 and 2 had a normal karyotype. No information are available about case 3 .

\section{Post-natal feedback}

In our review, a postnatal feedback with MRI and $\backslash$ or autopsies was performed/available in 57 cases (88\%), confirming prenatal diagnosis. What emerged from post-natal studies, is the alteration in aqueductal patency, together with midbrain tegmentum dysplasia, have been reported in postnatal studies $[1,8,10,17]$. Early aqueductal obstruction during fetal life could explain some of the supra-tentorial anomalies such as the hydrocephalus and the extreme corpus callosum thinning or interruption. The cause of this condition in RES is unknown.

In our experience, unfortunately, it was not possible to have postnatal data because in cases 1 and 2 the couple decided to terminate pregnancy in another institution and in case 3 the mother did not allow any further investigation on the stillborn child. Prenatal 
MRI of case 1 could not establish with certainty whether the aqueduct of Silvius was patent or not.

At birth, the clinical presentation as well as the prognosis is variable and seems to be related to the presence and severity of associated supra-tentorial anomalies [12-17]. In our review, we could not obtain much post-natal clinical information, since termination of pregnancy was decided in $91 \%$ of cases. The prognosis of the remaining pregnancies was poor in $85 \%$ of cases. Indeed, among the remaining 6 fetuses, 2 were stillborn, 2 died immediately after birth or in the neonatal period, while 1 died at 18 months of age.

Only Koprivek [28] reported a case of a prenatal diagnosis of partial isolated RES that at 12 months of age showed a slight delay in psychomotor development, mild hypotonia with normal cognitive development. Other [42] reported cases diagnosed postnatally, with significant neurologic symptoms such as irritability, poor balance, head rolling, abnormal eye movement, spasticity, dysarthria, strabismus, mild truncal ataxia, self-mutilation, and obsessivecompulsive disorder.

\section{Conclusions}

Prenatal diagnosis of RES still represents a challenge for maternal- fetal Medicine, since it presents rarely but with a severe prognosis. In our review, most of fetuses were referred to prenatal MRI for ventriculomegaly and suspected cerebellar hypoplasia, confirming that a careful evaluation of the PF is mandatory when a ventriculomegaly and a small cerebellum are seen at US, particularly when other CNS midline abnormalities are associated findings. Using US and MRI to its maximum capability, together with a multidisciplinary discussion may overcome this challenge, leading to the final diagnosis.

\section{References}

1. Truwit CL, Barkovich AJ, Shanahan R, Maroldo TV. MR imaging of rhombencephalosynapsis: report of three cases and review of the literature. AJNR Am J Neuroradiol. 1991; 12: 957-965.

2. Aydingo"z U", Cila A, Aktan G. Rhombencephalosynapsis associated with hand anomalies. Br J Radiol. 1997; 70: 764-766.

3. Litherland J, Ludlam A, Thomas N. Antenatal ultrasound diagnosis of cerebellar vermian agenesis in a case of rhombencephalosinapsis. J Clin Ultrasound. 1993; 21: 636-638.

4. Verri A, Uggetti C, Vallero E, Ceroni M, Federico A. Oral self-mutilation in a patient with rhombencephalosynapsis. J Intellect Disabil Res. 2000; 44: 86-90.

5. Obersteiner H. Ein Kleinhirn ohne Wurm: Arb Neurol Inst (Wien). 1914; 21 124-136.

6. Savolaine ER, Fadell RJ, Patel YP. Isolated rhombencephalosinapsis diagnosed by magnetic resonance imaging. Clin Imaging. 1991; 15: 125-129.

7. Toelle SP, Yalcinkaya C, Kocer N, Deonna T, Overweg-Plandsoen WC, Bast $\mathrm{T}$, et al. Rhombencephalosynapsis: clinical findings and neuroimaging in 9 children. Neuropediatrics. 2002; 33: 209-214.

8. Danon O, Elmaleh M, Boukobza B, Fohlen M, Hadjnacer K, Hassan M Rhombencephalosynapsis diagnosed in childhood: clinical and MRI findings. Magn Reson Imaging. 2000; 18: 99-101.

9. Montull C, Mercader JM, Peri J, Martínez Ferri M, Bonaventura I. Neuroradiological and clinical findings in rhombencephalosynapsis. Neuroradiology. 2000; 42: 272-274.

10. Utsunomiya H, Takano K, Ogasawara T, Hashimoto T, Fukushima T,
Okazaki M. Rhomboencephalosynapsis: cerebellar embryogenesis. AJNR Am J Neuroradiol. 1998; 19: 547-549.

11. Bekiesińska-Figatowska M, Jurkiewicz E, Szkudlińska-Pawlak S, Malczyk K, Nowak K. Rhombencephalosynapsis - isolated anomaly or complex malformation? Pol J Radiol. 2012; 77: 35-38.

12. Altman NR, Naidich TP, Braffman BH. Posterior fossa malformations. AJNR Am J Neuroradiol. 1992; 13: 691-724.

13. Demaerel P, Kendall BE, Wilms G, Halpin SF, Casaer P, Baert AL. Uncommon posterior cranial fossa anomalies: MRI with clinical correlation. Neuroradiology. 1995; 37: 72-76.

14. Simmons G, Damiano TR, Truwit CL. MRI and clinical findings in rhombencephalosynapsis. J Comput Assist Tomogr. 1993; 17: 211-214.

15. Romanengo M, Tortori-Donati P, Di Rocco M. Rhombencephalosynapsis with facial anomalies and probable autosomal recessive inheritance: a case report. Clin Genet. 1997; 52: 184-186.

16. Barkovich AJ. Pediatric neuroimaging; in: Norman D, ed. Contemporary Neuroimaging. New York: Raven. 1990: 113-115

17. Pasquier L, Marcorelles P, Loget P, Pelluard F, Carles D, Perez MJ, et al. Rhombencephalosynapsis and related anomalies: a neuropathological study of 40 fetal cases. Acta Neuropathol. 2009; 117: 185-200.

18. Sener RN. Rhombencephalosynapsis associated with Dandy-Walker malformation. Journal of Neuroimaging. 2007; 17: 355-357.

19. Sidman RL, Rakic P. Development of the human central nervous system. In: Haymaker W, Adams RD, eds. Histology and Histopathology of the Nervous System. Springfield: Thomas. 1982: 3-145.

20. Michaud J, Mizrahi EM, Urich $\mathrm{H}$. Agenesis of the vermis with fusion of the cerebellar hemispheres, septo-optic dysplasia and associated anomalies. Report of a case. Acta Neuropathol (Berl). 1982; 56: 161-166.

21. Paladini D, Volpe P. Ultrasound of congenital fetal anomalies. 2014 CRC Press.

22. Ten Donkelaar HJ, Lammens M, Hori A. Clinical Neuroembryology. 2014 Springer.

23. Robinson AJ. Inferior vermian hypoplasia-preconception, misconception. Ultrasound Obstet Gynecol. 2014; 43: 123-136

24. Yachnis AT. Rhombencephalosynapsis with massive hydrocephalus: case report and pathogenetic considerations. Acta Neuropathol (Berl). 2000; 103: 301-304.

25. Millen KJ, Millonig JH, Hatten ME. The mouse Dreher gene Lmx1a controls formation of the roof plate in the vertebrate CNS. Nature. 2000; 403: 764-769.

26. McAuliffe F, Chitayat D, Halliday W, Keating S, Shah V, Fink M, et al. Rhombencephalosynapsis: prenatal imaging and autopsy findings. Ultrasound Obstet Gynecol. 2008; 31: 542-548.

27. Izzo G, Conte G, Cesaretti C, Parazzini C, Bulfamante G, Righini A. Prenatal Magnetic Resonance Imaging of Atypical Partial Rhombencephalosynapsis with Involvement of the Anterior Vermis: Two Case Reports. Neuropediatrics. 2015; 46: 416-419.

28. Koprivsek KM, Novakov-Mikic AS, Lucic MA, Kozic DB, Till VE, Belopavlovic Z. Partial rhombencephalosynapsis: prenatal MR imaging diagnosis and postnatal follow up. Acta Neurol Belg. 2011; 111: 157-159.

29. Arisoy R, Erdogdu E, Pekin O, Tugrul S, Aydin H, Yorganci C. A rare case of rhombencephalosynapsis and prenatal diagnosis. J Obstet Gynaecol. 2016; 36: 839-841.

30. Poretti A, Boltshauser E. Fetal Diagnosis of Rhombencephalosynapsis. Neuropediatrics. 2015; 46: 357-358.

31. Napolitano M, Righini A, Zirpoli S, Rustico M, Nicolini U, Triulzi F. Prenatal magnetic resonance imaging of rhombencephalosynapsis and associated brain anomalies: report of 3 cases. J Comput Assist Tomogr. 2004; 28: 762765.

32. di Vera E, Liberati M, Celentano C, Calabrese G, Guanciali-Franchi PE, 
Morizio E, et al. Rhombencephalosynapsis in a severely polymalformed fetus with non-mosaic tetrasomy $9 p$, in intracytoplasmic-sperm-injection pregnancy. J Assist Reprod Genet. 2008; 25: 577-580.

33. Litherland J, Ludlam A, Thomas N. Antenatal ultrasound diagnosis of cerebellar vermian agenesis in a case of rhombencephalosynapsis. J Clin Ultrasound. 1993; 21: 636-638.

34. Leibovitz Z, Shkolnik C, Haratz KK, Malinger G, Shapiro I, Lerman-Sagie T. Assessment of fetal midbrain and hindbrain in mid-sagittal cranial plane by three-dimensional multiplanar sonography. Part 2: application of nomograms to fetuses with posterior fossa malformations. Ultrasound Obstet Gynecol. 2014; 44: 581-587.

35. Bernardo S, Vinci V, Saldari M, Servadei F, Silvestri E, Giancotti A, et al. Dandy-Walker Malformation: is the 'tail sign' the key sign? Prenat Diagn. 2015; 35: 1358-1364.

36. Cagneaux M, Vasiljevic A, Massoud M, Allias F, Massardier J, Gaucherand $\mathrm{P}$, et al. Severe second-trimester obstructive ventriculomegaly related to disorders of diencephalic, mesencephalic and rhombencephalic differentiation. Ultrasound Obstet Gynecol. 2013; 42: 596-602.

37. De Keersmaecker B, Ramaekers P, Claus F, Witters I, Ortibus E, Naulaers $\mathrm{G}$, et al. Outcome of 12 antenatally diagnosed fetal arachnoid cysts: case series and review of the literature. Eur J Paediatr Neurol. 2015; 19: 114-121.

38. Blazer S, Berant M, Sujov PO, Zimmer EZ, Bronshtein M. Prenatal sonographic diagnosis of vermal agenesis. Prenat Diagn. 1997; 17: 907-911.
39. Bromley B, Nadel AS, Pauker S, Estrogg JA, Benacerraf BR. Closure of the cerebellar vermis: evaluation with second trimester US. Radiology. 1994; 193: 761-763.

40. Elliott R, Harter DH. Rhombencephalosynapsis associated with autosomal dominant polycystic kidney disease Type 1. J Neurosurg Pediatr. 2008; 2: 435-437.

41. Tully HM, Dempsey JC, Ishak GE, Adam MP, Curry CJ, Sanchez-Lara P, et al. Beyond Gómez-López-Hernández syndrome: recurring phenotypic themes in rhombencephalosynapsis. Am J Med Genet A. 2012; 158A: 23932406.

42. Paprocka J, Jamroz E, Ścieszka E, Kluczewska E. Isolated rhomboencephalosynapsis - a rare cerebellar anomaly. Pol J Radiol. 2012; 77: 47-49.

43. Ghymers D, Hemann B, Distèche C, Frederic J. Tetrasomie partielle du chromosome 9 à l'ètat mosaique, chez un enfant porteur de malformations multiples. Hum Genet. 1973; 20: 273-282.

44. Dhandha S, Hogge WA, Surti U, McPherson E. Three cases of tetrasomy 9p. Am J Med Genet. 2002; 113: 375-380.

45. Chen CP, Chang TY, Shih JC, Lin SP, Lin CJ, Wang W, et al. Prenatal diagnosis of dandy-Walker malformation and ventriculomegaly associated with partial trisomy $9 p$ and distal $12 p$ deletion. Prenat Diagn. 2002; 12: 10631065 